\title{
Imaging Live Uterine Tissue Modulation Using Confocal Microscopy
}

\author{
S.M.B. Obayomi ${ }^{1}$, S. Peck ${ }^{1}$ and D.P. Baluch ${ }^{1}$ \\ 1. School of Life Sciences, Arizona State University, Tempe, Arizona 85287 USA
}

Pregnancy and childbirth are both natural occurring events, but still little is known about the signaling mechanisms that induce contractions. Throughout the world, premature labor occurs in $12 \%$ of all pregnancies with 36\% of infant deaths resulting from preterm related causes [1]. Even though the cause of preterm labor can vary, understanding alternative signaling pathways which affect muscle contraction could provide additional treatment options in stopping premature labor. The uterus is composed of smooth muscle which is innervated with a plexus of nerves that cover the muscle fibers. Smooth muscle can be stimulated or modulated by many sources such as neurotransmitters [dopamine], hormones [estrogen], peptides [oxytocin] and amines. In this study, we are focusing on the biogenic monoamine tyramine which is produced in the tyrosine catecholamine biosynthesis pathway. Tyramine is known to be associated with peripheral vasoconstriction, increased cardiac output, increased respiration, elevated blood glucose and the release of norepinephrine. Our research has found tyramine, and its specific receptor TAAR1 [2], to be localized within mouse uterus and that this monoamine can induce uterine contractions at levels similar to oxytocin.

Research that focuses on reproduction commonly use techniques involving immunohistochemistry or histological staining to identify localization of key modulators and receptors or they observe general morphology in fixed whole or sectioned tissue [Fig. 1]. In studies involving muscle tissue, force transduction measurements allow the researcher to quantify differences in contractile force. These methods can be used with the addition of agonists and antagonists which help decipher the mechanisms that may modulate the contraction. A drawback of these methods is that muscle contraction is a complex system that relies on feedback mechanisms and without observing the tissue in real time, the researcher would be making interpretations based on random snapshots in time. To overcome this limitation, we have developed an imaging technique that can observe muscle signaling dynamics in real time.

This protocol was approved through the IACUC protocol 15-1388T at Arizona State University and the mouse uterine tissues were derived from GFP LifeAct transgenic mice [3]. These mice contain a transgene encoding a 17-amino acid peptide called LifeAct, which binds F-actin. This peptide is coexpressed with GFP which enables the actin cytoskeleton to be imaged both in vivo and in vitro. Actin is a key component of muscle tissue and this transgenic model allows researchers the ability to visualize muscle contractions. Combining this tissue with the TAAR1 receptor antibody conjugated to a fluorescent probe [antibody labeling kit, Thermo Fisher, \#A20186] is a tool that can be used to visualize the actual response and timing of a receptor mediated event. Imaging was conducted using a dipping lens on a Leica SP5 confocal microscope which allowed the tissue to continue normal peristaltic contractile activity. Time-lapse imaging was set up to allow for a period of non-treatment for 5 minutes followed by a 15-minute response time after treatment. Experiments using various stimulators demonstrated the dynamic response of TAAR1 receptor recruitment especially in response to catecholamines such as dopamine which causes muscle relaxation (Fig.2). This imaging technique has proved to be very useful in further understanding the actions induced by the use of signaling modulators and correlating the results found using standard techniques on fixed tissues. 


\section{References}

[1.] C. Ferre, et.al. (2016). Effects of Maternal Age and Age-Specific Preterm Birth Rates on Overall Preterm Birth Rates. MMWR, 65(43) 1181-84.

[2.] V.M. Lam, et.al. (2015). Discovery of trace amine-associated receptor 1 ligands by molecular docking screening against a homology model. Med. Chem. Commun. 6, 2216-23.

[3.] J. Riedl, et.al. (2008). Lifeact: a versatile marker to visualize F-actin. Nat Methods 5(7), 605-7.

[4.] We acknowledge the School of Life Sciences at ASU for their generous graduate research award funding.

\section{Figures}
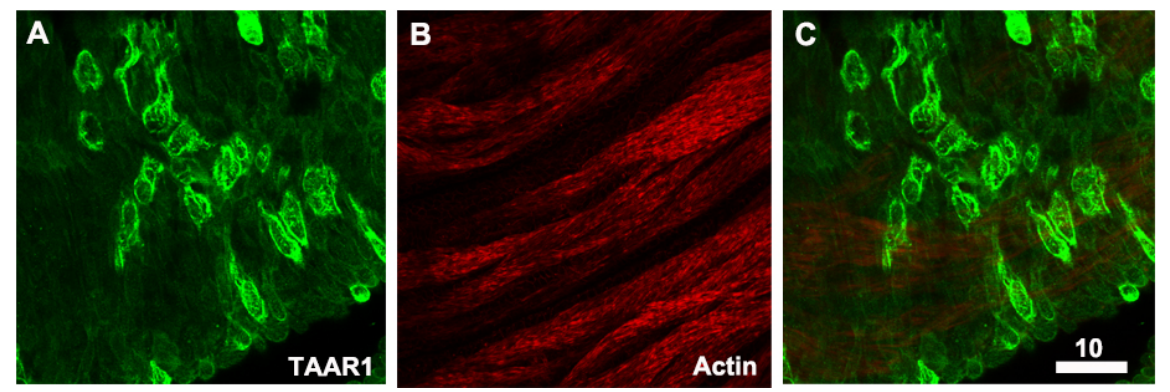

Fig. 1. Fixed pseudo-pregnant mouse uterine muscle labeled with an antibody against A) the tyramine specific TAAR1 receptor (green) and B) Phalloidin (red) to highlight the smooth muscle. Image C is an overlay of images A and B. Scale bar is $10 \mu \mathrm{m}$.

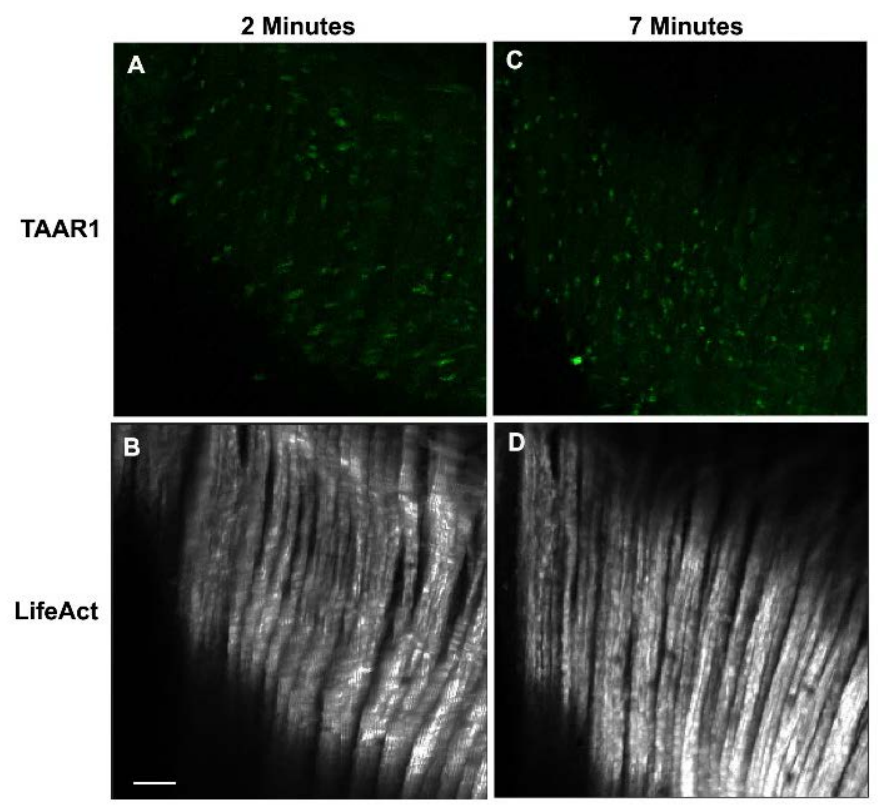

Fig. 2. Live uterine muscle from a transgenic GFP LifeAct mouse is used to study the mechanisms which regulate contractions. Actin filaments within the muscle are pictured in grayscale (B and D) and the TAAR1 receptor, conjugated to Alexa 647 fluorophore, is green (A and $\mathrm{C}$ ). The tissue is treated with dopamine at a 5 minute timepoint which causes the tissue to relax. This generates a response to recruit additional TAAR1 receptor to the membrane [C] to restore the peristaltic contractile activity. These images are snapshots from a time lapse video. Scale bar is $10 \mu \mathrm{m}$. 